BNL-112603-2016-JA

\title{
Exciton Correlations in Intramolecular Singlet Fission
}

Samuel N. Sanders, Elango Kumarasamy, Andrew B. Pun, Kannatassen Appavoo, Michael L. Steigerwald, Luis M. Campos and Matthew Y. Sfeir

Submitted to the Journal of the American Chemical Society

May 2016

Center for Functional Nanomaterials

Brookhaven National Laboratory

U.S. Department of Energy
USDOE Office of Science (SC),
Basic Energy Sciences (SC-22)

Notice: This manuscript has been authored by employees of Brookhaven Science Associates, LLC under Contract No. DE-SC0012704 with the U.S. Department of Energy. The publisher by accepting the manuscript for publication acknowledges that the United States Government retains a non-exclusive, paid-up, irrevocable, world-wide license to publish or reproduce the published form of this manuscript, or allow others to do so, for United States Government purposes. 


\section{DISCLAIMER}

This report was prepared as an account of work sponsored by an agency of the United States Government. Neither the United States Government nor any agency thereof, nor any of their employees, nor any of their contractors, subcontractors, or their employees, makes any warranty, express or implied, or assumes any legal liability or responsibility for the accuracy, completeness, or any third party's use or the results of such use of any information, apparatus, product, or process disclosed, or represents that its use would not infringe privately owned rights. Reference herein to any specific commercial product, process, or service by trade name, trademark, manufacturer, or otherwise, does not necessarily constitute or imply its endorsement, recommendation, or favoring by the United States Government or any agency thereof or its contractors or subcontractors. The views and opinions of authors expressed herein do not necessarily state or reflect those of the United States Government or any agency thereof. 


\title{
Exciton Correlations in Intramolecular Singlet Fission
}

\author{
Samuel N. Sanders', Elango Kumarasamy ${ }^{\dagger}$, Andrew B. Pun ${ }^{\dagger}$, Kannatassen Appavoo ${ }^{\ddagger}$, Michael L. \\ Steigerwald ${ }^{\dagger}$, Luis M. Campos ${ }^{\star \star}$, Matthew Y. Sfeir ${ }^{\star *}$ \\ † Department of Chemistry, Columbia University, New York, New York 10027, United States \\ ${ }^{\ddagger}$ Center for Functional Nanomaterials, Brookhaven National Laboratory, Upton, New York 11973, United States
}

\begin{abstract}
We have synthesized a series of asymmetric pentacene-tetracene heterodimers with a variable-length conjugated bridge that undergo fast and efficient intramolecular singlet fission (iSF). These compounds have distinct singlet and triplet energies, which allow us to study the spatial dynamics of excitons during the iSF process, including the significant role of exciton correlations in promoting triplet pair generation and recombination. We demonstrate that the primary photoexcitations in conjugated dimers are delocalized singlets that enable fast and efficient iSF. However, in these asymmetric dimers, the singlet becomes more localized on the lower energy unit as the length of the bridge is increased, slowing down iSF relative to analogous symmetric dimers. We resolve the recombination kinetics of the inequivalent triplets produced via iSF, and find that they primarily decay via concerted processes. By identifying different decay channels, including delayed fluorescence via triplet-triplet annihilation, we can separate transient species corresponding to both correlated triplet pairs and uncorrelated triplets. Recombination of the triplet pair proceeds rapidly despite our experimental and theoretical demonstration that individual triplets are highly localized and unable to be transported across the conjugated linker. In this class of compounds, the rate of formation and yield of uncorrelated triplets increases with bridge length. Overall, these constrained, asymmetric systems provide a unique platform to isolate and study transient species essential for singlet fission, which are otherwise difficult to observe in symmetric dimers or condensed phases.
\end{abstract}

\section{INTRODUCTION}

Multiple exciton generation from a single photon has tremendous potential for technological applications and has generated wide interest in organic singlet exciton fission (SF) compounds. ${ }^{1-9} \mathrm{~A}$ few basic requirements for SF have been developed from advanced theoretical and spectroscopic studies of molecular crystals, which can be used to set the foundation for materials design and fuel the development of next generation devices. ${ }^{10-22}$ For example, it has been well established that materials only undergo singlet fission when strong interchromophore electronic interactions are present, extending the spatial distribution of the singlet state over neighboring molecules. ${ }^{23-26}$ Additionally, the materials must meet the energy conservation requirement - the singlet state must be greater than or equal to the triplet pair state. ${ }^{5}$ Furthermore, it is understood that in molecular crystals, uncorrelated triplets rapidly form at room temperature from a multiexciton state and diffuse apart, though non-geminate triplettriplet annihilation can repopulate the singlet state when the energetics of the system permit it. ${ }^{12,13,27-31}$

While the initial and final states of SF have been widely studied, details of the dynamics that occur during singlet fission, e.g., between the singlet and correlated multiexciton (triplet pair) state, have been more difficult to discern. This uncertainty has stemmed from both the short lifetime of the correlated state in condensed phase systems and the similarity of the correlated and uncorrelated triplet pair spectral signatures. ${ }^{14,32-34}$ These issues have led to the development of artificial systems designed to isolate exciton correlations, by either slowing down the dynamics of singlet fission, using high concentration solutions for example, or by introducing distinct spectral signatures for singlet fission, as in doped single-crystals. ${ }^{35-37}$ Still, there is a lack of a comprehensive understanding of the correlated triplet pair exciton, including a detailed understanding of its formation and decay dynamics.

The recent discovery of efficient intramolecular singlet fission (iSF) materials based on molecular dimers and polymers has expanded the quantity and variety of materials that undergo singlet fission..$^{9,38-42}$ They also offer a unique platform to study the dynamical evolution of multiexciton states since the system can be constrained such that exactly two triplet sites exist on the molecule. This restriction prevents separation via diffusion, ensuring that all bimolecular recombination processes are geminate. Therefore, this system allows us to identify distinct dynamical processes attributable to a triplet pair. Furthermore, in oligoacene dimers, we can systematically tune the interchromophore interactions, e.g., their proximity, connectivity, or planarity to dramatically modify the triplet pair generation and recombination kinetics. ${ }^{41,43}$

Here, we take advantage of differences in the optical characteristics between tetracene and pentacene to study the exciton dynamics or iSF in a family of pentacenetetracene heterodimers (Figure 1). The asymmetry of these dimers results from inequivalent singlet and triplet energies in the parent monomers, yielding distinct spectral features associated with excitation of the individual chromophores in the dimer. ${ }^{43}$ Critically, this allows us to 
monitor directly the spatial dynamics of the singlet exciton, as well as both the triplet pair states produced via singlet fission and the individual triplet states populated via sensitization. By varying the bridge length in the dimer, we can directly show how interchromophore separation affects the triplet formation and relaxation processes.

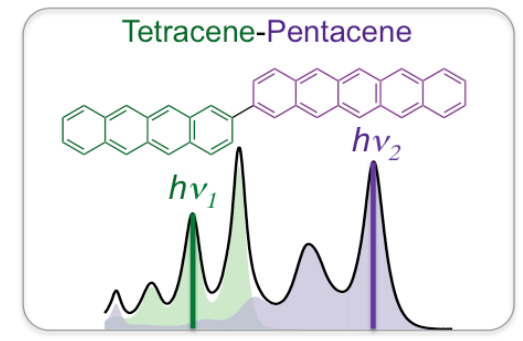

Proposed Mechanisms for iSF Localized singlet? Delocalized singlet?

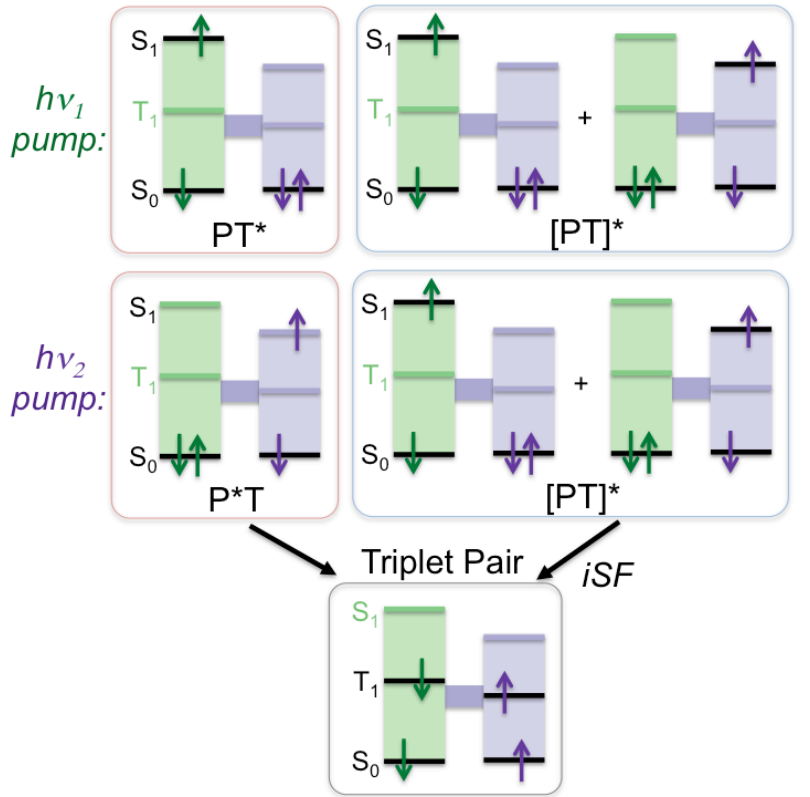

Figure 1. (Top) Representation of the absorption spectra of pentacene (purple) and tetracene (green) chromophores, along with their summed absorption (black line). (Middle) Depiction of the competing paths for intramolecular singlet fission based on localized (left) and delocalized (right) singlet states. Asymmetric dimers allow us to demonstrate the delocalization of the singlet exciton in iSF compounds. (Bottom) The product of singlet fission is a triplet pair, with one triplet localized on each chromophore.

The spatial resolution provided by asymmetric dimers is critical for understanding singlet fission, including the role of exciton correlations. For example, while varying the connectivity in pentacene dimers has yielded valuable information about the kinetics of iSF, there is still considerable debate surrounding the role of singlet exciton delocalization in promoting singlet fission. The understanding of the nature of the photoexcited singlet is of particular interest because it has been suggested that delocalization may be important for promoting rapid singlet fission, and dimers are an ideal model system to further examine this possibility. ${ }^{44}$ In a study on ethynylbenzeneseparated dimers, the authors propose that the initial photoexcited singlet is localized on one of the two pentacenes in the dimer $\left(\mathrm{P}^{*} \mathrm{P}\right)$. From the time-resolved transient absorption spectra, an apparent rise of the bleach during singlet fission is reported, which is interpreted as fission from a highly localized singlet state, in contrast to what is observed in condensed phase systems. ${ }^{42,45}$ On the other hand, two other studies, including our own, report pentacene dimers in which the singlet exciton is initially distributed over both monomers $\left([\mathrm{PP}]^{*}\right)$ and demonstrate that overlapping excited state absorption signals associated with the singlet can complicate the single-wavelength kinetics. ${ }^{40,41}$ These two competing mechanisms are shown schematically in Figure 1 for the asymmetric pentacenetetracene (PT) dimers studied here.

Here, we use the term delocalized singlet to describe the situation in which the optically bright state is a linear combination of two locally excited states: $[\mathrm{PT}]^{*}=\left[a \mathrm{PT}^{*}+\right.$ $\left.b \mathrm{P}^{*} \mathrm{~T}\right]$. This picture of a linear combination of locally excited configurations is an example of the mixing of alternatives that is familiar to organic chemistry as the interaction of valence-bond resonance structures. As Pauling originally described ${ }^{46}$, the closer in energy the two alternative structures are, the more important the mixing; in bipentacenes the two alternatives are degenerate while in the present case the two, $\mathrm{PT}^{*}$ and $\mathrm{P}^{*} \mathrm{~T}$, are energetically distinct, albeit nearly degenerate. This picture of locally excited monomer state mixing is supported by multireference electronic structure calculations on both noncovalent ${ }^{15,47}$ and covalent pentacene dimers ${ }^{48}$ and by the experimental absorption spectra of bipentacence ${ }^{41}$ and pentacene-tetracene heterodimers (Figure 2), in which the monomer and dimer singlet energies differ by only $30 \mathrm{meV}$. Since there is no systematic dependence on spacer length, the effects are likely related to a change in the effective dielectric screening due to the proximity of an additional chromophore and its associated solubilizing groups. We note that this "delocalization" is what Pauling referred to as resonance delocalization, and it should not be confused with simple orbital delocalization that results in a particle-in-a-box picture. The latter would dramatically shift the energy of the dimer relative to the monomer, the former yields comparatively minor energy consequences. Throughout this manuscript, we use the term delocalization to refer to this resonance delocalization: the linear combination of locally excited states on either the pentacene or tetracene monomer.

The distinct pentacene and tetracene absorption features provide a clear test of a delocalized singlet, in which case an instantaneous bleach of the tetracene spectral features would result when pumping lower energy pentacene absorption features. In contrast to bipentacene dimers, this method is direct and does not rely on resolving complex overlapping transient signals. Similarly, the distinguishability of the individual components in PT allows for direct study of their dynamical evolution, which is not possible in symmetric molecules. This includes the ability to differentiate between concerted and sequential triplet pair decay.

Here, we address these fundamental, unanswered questions by unambiguously characterizing the intrinsic 
excited state dynamics, from triplet pair formation and recombination, with exquisite spatial and spectral resolution. We use a series of PT heterodimers containing phenylene spacers between the chromophores to show that the photogenerated singlets are delocalized over both monomers $\left([\mathrm{PT}]^{*}\right)$ and that triplet pair formation depends, to some extent, on the extent of singlet delocalization. Furthermore, our study indicates that iSF primarily yields correlated triplet pairs, which exhibit concerted decay processes. This includes a triplet-triplet annihilation process that results in delayed fluorescence, but also a non-radiative internal conversion mechanism, which is distinct from individual triplet decay processes. These decay processes occur rapidly despite the fact that individual triplets are not able to diffuse across the bridge, as determined via triplet sensitization experiments. In bridged compounds, a minority population of uncorrelated triplets are formed with a yield that increases with bridge length.

\section{RESULTS AND DISCUSSION \\ Synthesis}

In order to investigate singlet fission in heterodimers, the pentacene-tetracene heterodimers shown in Figure 2 were synthesized via a modular Suzuki coupling strategy that we have adopted for the coupling of various acene building blocks (SI for details). ${ }^{41}$ The compounds are labeled as PTn, where P refers to pentacene, $T$ refers to tetracene, and $\mathrm{n}$ refers to the number of phenylene spacers in the linker series, where $\mathrm{n}=0,1,2$. The inclusion of triisopropylsilyl acetylene (TIPS) groups renders these heterodimers soluble and stable in solution. ${ }^{49-51}$

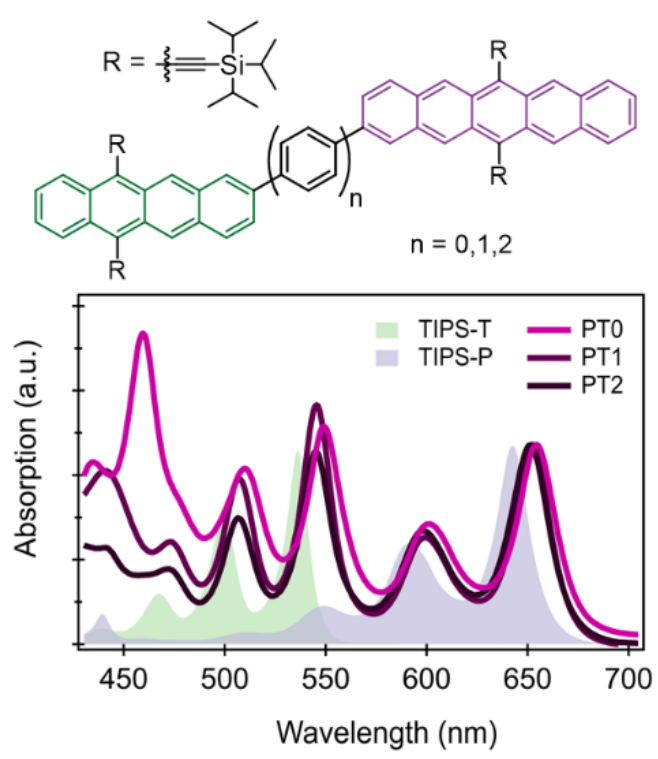

Figure 2. Chemical structure (top) and steady-state absorption spectra (bottom) of the PTo, PT1, and PT2 compounds $(\mathrm{n}=\mathrm{o}, 1$ or 2 ) along with TIPS-tetracene and TIPS-pentacene. Absorption spectra are taken in chloroform and normalized at the lowest energy absorption feature.

\section{Absorption Features}

The steady state absorption of the PTn heterodimers shows the characteristic absorption features of both monomers, with the prominent low-energy TIPS-pentacene peak at $\sim 660 \mathrm{~nm}$ and the tetracene peak at $\sim 550 \mathrm{~nm}$ (Figure 2). The relative intensity of the pentacene and tetracene features in the heterodimer spectrum varies among the different compounds, but not systematically with spacer length. Compared to our model absorption (direct sum of monomers) in Figure 1, we observe a small redshift relative to the monomer of both pentacene and tetracene features. The high energy feature observed in PTo is similar to BPo, our previously reported bipentacene, shifted by $\sim 40 \mathrm{~nm}$. This feature is specific to directly linked dimers, and does not correspond to a peak in the parent monomers. ${ }^{41}$

\section{Singlet Delocalization and Exciton Fission}
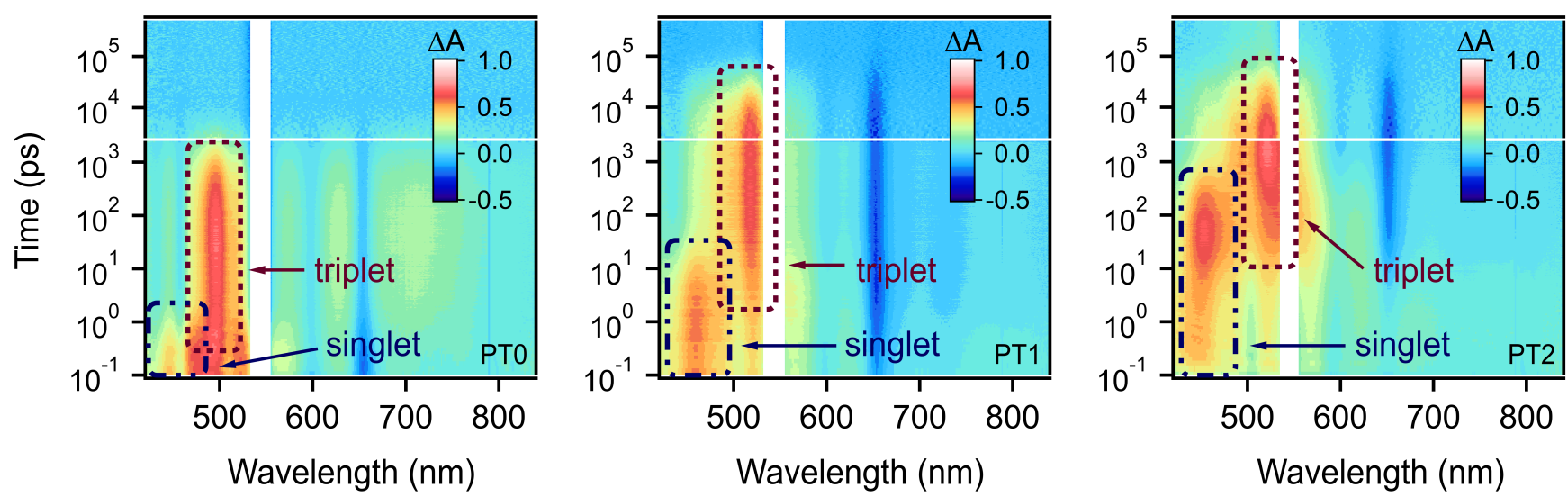

Figure 3. Transient absorption spectra of PTo, PT1 and PT2 (left to right), excited at $545 \mathrm{~nm}\left(\sim 25 \mu \mathrm{J} / \mathrm{cm}^{2}\right)$ in chloroform. The most prominent singlet (dot dash) and triplet (dash) excited state absorption features are outlined for clarity. 
We use broadband transient absorption spectroscopy to understand the exciton dynamics in these molecules. Qualitatively, the dynamics of PTn compounds are similar to those observed in their bipentacene analogs (here referred to as BPn, with $\mathrm{n}$ similarly representing the number of phenylene spacers), with a photoexcited singlet exciton rapidly decaying into a triplet pair in dilute solution, consistent with iSF (Figure 3). The assignment of the singlet and triplet states is accomplished similar to previous work from our group and others for pentacene dimers. ${ }^{40-42,52}$ A brief but thorough discussion of the assignments is found below, with a more extensive discussion in the SI. We assign the singlet excited state absorption features using the correspondence of their temporal decay to the prompt fluorescence signal. We assign the triplet excited state absorption features by comparing the transient spectra of the triplet pair (generated by direct photoexcitation followed by singlet fission) to an individual triplet generated by sensitization. Furthermore, we find that the triplet spectra in these compounds are nearly identical to those in symmetric bipentacene dimers, due to the much large extinction coefficient of a pentacene triplet relative to a tetracene triplet. ${ }^{35,53}$ Finally, we note that the dynamics are concentration independent, ruling out excimertype singlet deactivation processes (SI).

In contrast to homodimers, the compounds have two unique ground state bleach signals associated with tetracene $(\sim 550 \mathrm{~nm})$ and pentacene $(\sim 660 \mathrm{~nm})$ that can be used to track the relative occupation of excitons on each chromophore. Features associated with the triplet pair produced by iSF are dominated by the pentacene $T_{1} \rightarrow T_{3}$ triplet excited state absorption ( $500-520 \mathrm{~nm}$, dash line in Figure 3) due to the much larger triplet absorption crosssection of TIPS-pentacene relative to TIPS-tetracene. ${ }^{35,53}$ As observed in homodimers, the singlet fission rate slows down with increasing chromophore separation (larger number of phenyl ring spacers), with time constants of o.83 ps, 18.3 ps, and 640 ps for PTo, PT1, and PT2, respectively. There is no indication of a parasitic process that would affect the iSF yield. In other words, the rates of singlet decay and triplet formation are directly correlated, and the yields are determined only by the kinetic competition between iSF and the intrinsic monomer decay processes $(\sim 13 \mathrm{~ns}))^{35,39,41,53}$

We find that delocalized singlet excitons are essential for fast and efficient singlet fission. In PTn, the photoexcited singlet exciton contains a signature of both the tetracene and pentacene ground state bleach, even when selectively pumping the absorption features associated with the pentacene monomer. This can be clearly seen by inspection of Figure 4a (top panel), which shows the isolated transient spectra corresponding to the singlet, solved by global analysis methods (SI). ${ }^{54}$ Here, a pump pulse at $600 \mathrm{~nm}$, resonant with a vibrationally excited $S_{1}$ state of pentacene, results in an impulsive bleach of tetracene absorption features at $550 \mathrm{~nm}$. Indistinguishable SF dynamics are observed for direct resonant pumping of the pentacene absorption at $660 \mathrm{~nm}$ (SI). The bleach of both chromophores implies a singlet exciton that is delocalized to some extent over the whole molecule.
Interestingly, in $\mathrm{PT}_{1}$ and $\mathrm{PT}_{2}$ (Figure 4a), the relative magnitude of the tetracene bleach is reduced when selectively pumping pentacene-associated absorption features as the spacer length is increased. The reduced contribution of the tetracene ground state bleach signals suggests that the singlet wavefunction contains a larger relative fraction of a localized pentacene excitation. This conclusion is supported by density functional theory calculations (SI) of the LUMO of these molecules as a function of linker length. While more sophisticated electronic structure methods are necessary to accurately describe the electron correlations these molecules, these calculations illustrate the general trend that the singlet wavefunction starts to resemble a localized pentacene excitation as the length of the bridge increases. Because the pentacene monomer has a smaller singlet energy than tetracene, it is reasonable to expect some preference of the singlet exciton for the pentacene subunit as the electronic coupling is reduced. Following iSF, the isolated triplet pair spectra show the prominent ground state bleach features of pentacene and tetracene in similar magnitude (bottom panel in Figure 4a), as is expected for a triplet pair state with one triplet on each chromophore.

Exciting PT1 (SI) or PT2 (Figure 4b) with a $545 \mathrm{~nm}$ pump pulse that is resonant with the tetracene component results in a hot singlet state, which shows an enhanced tetracene ground state bleach signal. The hot singlet state cools rapidly with time constants of $\sim 1$ ps in PT1 and $\sim 12$ ps in PT2 to a state nearly identical to the singlet populated under pentacene resonant pumping conditions. During cooling, the relative intensity of the pentacene ground state bleach increases relative to the tetracene ground state bleach features. As cooling in each molecule is much faster than the corresponding overall singlet lifetime, the population and subsequent cooling of this hot exciton has a negligible effect on the overall iSF time constant $(\sim 19 \mathrm{ps}$ in PT1 and $\sim 600 \mathrm{ps}$ in PT2 with 545 nm pump).

The experimentally observed trend towards a more localized singlet (reduced tetracene ground state bleach signal) as the spacer length increases may have relevance to the observed SF rates. Similar SF rates are observed for PTo and $\mathrm{PT}_{1}$ as compared to $\mathrm{BPo}$ and $\mathrm{BP} 1$, respectively. However, in $\mathrm{PT}_{2}$, singlet fission is considerably slower (640 ps time constant) than $\mathrm{BP}_{2}$ (220 ps time constant). Using a similar electronic structure calculation for symmetric BPn as for PTn compounds, we find that the degeneracy of the monomers results in a more delocalized singlet for similar linker lengths. ${ }^{41}$ This result suggests that singlet exciton delocalization may be an important or even essential design principle for iSF. 

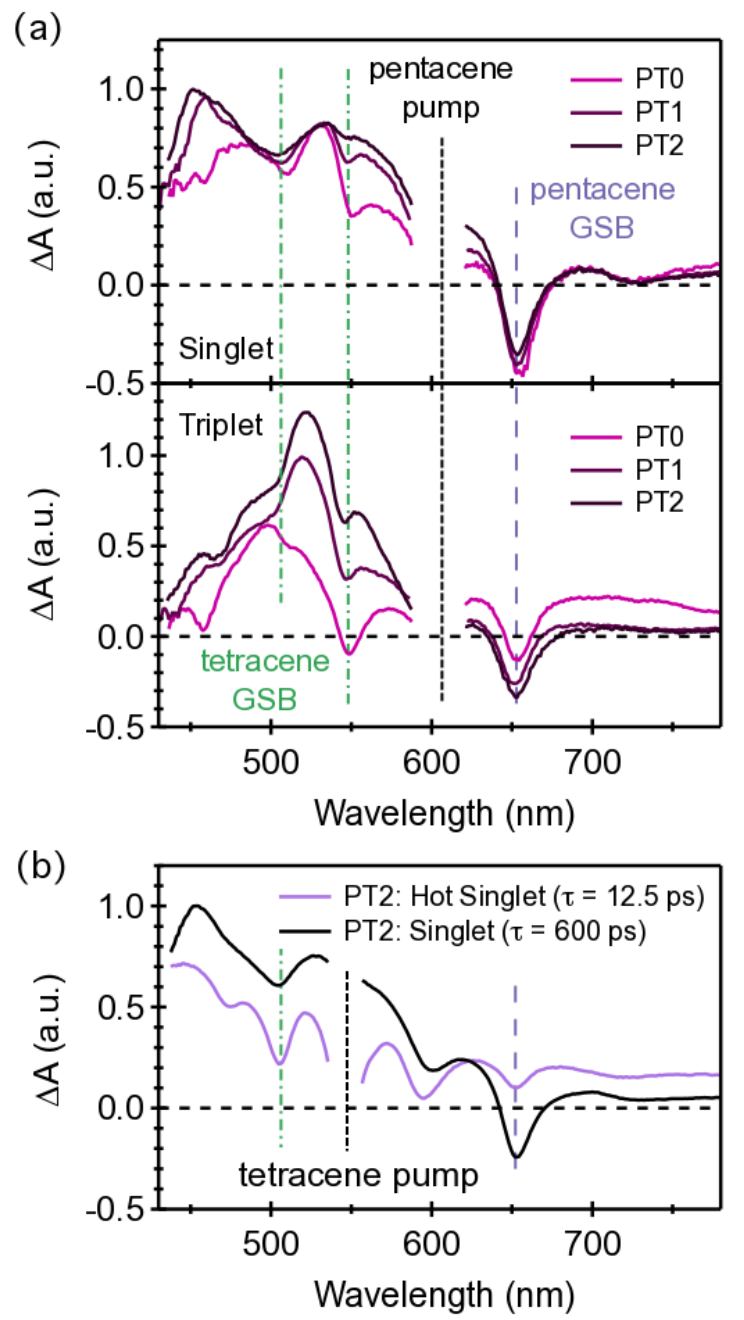

Figure 4. (a) Singlet and triplet pair excited absorption spectra from global analysis show that both the pentacene (purple dashed line) and tetracene (green dashed line) ground state bleach are present before (in unequal proportions) and after iSF (in similar proportions) when selectively pumping the low energy pentacene absorption features. (b) When a tetracene absorption peak is resonantly excited, an additional hot exciton state is observed that cools in 12.5 ps. This relaxed singlet state is nearly identical to the state observed when resonantly pumping pentacene, and subsequently undergoes iSF.

\section{Sensitized Triplet Dynamics}

The asymmetric oligoacene dimers also allow us to track the different recombination processes associated with the two inequivalent triplet sites. To this end, we utilize sensitization experiments, in which pentacene and tetracene triplets are individually populated via collisional transfer from a triplet donor (anthracene) in excess concentration (SI). Because anthracene can transfer an individual triplet to pentacene or tetracene with similar probability, we observe a roughly even mixture of pentacene and tetracene triplets after sensitization, as evidenced by the similar ground state bleach magnitudes for pentacene and tetracene (Figure 5).
We find that the triplets are not mobile, but instead remain localized on one half of the dimer. These localized, individual triplets decay with different time constants depending on whether the tetracene or pentacene sub-units are populated. Interestingly, we do not observe triplet transfer within a single heterodimer, e.g., from the higher energy tetracene triplet state to the lower energy pentacene triplet. This process would result in the exclusive population of pentacene triplets at later times. However, we observe the continued presence of both pentacene and tetracene ground state bleach signals at very long timescales. In fact, at long times, the tetracene ground state bleach actually increases relative to the pentacene ground state bleach (Figure 5) because tetracene triplets exhibit longer triplet lifetimes than pentacene triplets (SI) in agreement with the energy gap law. ${ }^{55-57}$ These decay dynamics indicate that both inter- and intramolecular triplet transport from tetracene to pentacene is slow compared to recombination. Presumably, the lack of triplet energy transfer is due to the minimal wave function overlap between the pentacene and tetracene triplet, which are both highly localized due to favorable exchange energy interactions, precluding Dexter energy transfer (further details in SI). ${ }^{58}$
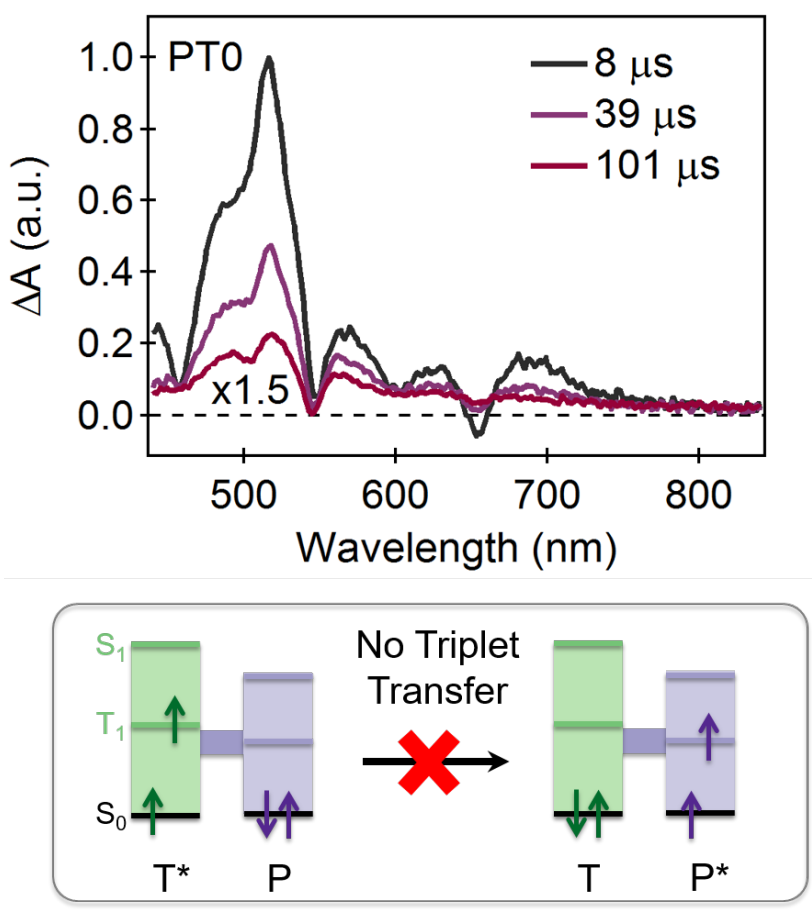

Figure 5. Representative sensitization data showing similar weights of GSB on pentacene and tetracene, and the lack of triplet transfer, even at long times

\section{Electronic Correlations in Triplet Pairs}

Since the ensemble of molecules contains an even population distribution of $T_{1}$ in both pentacene and tetracene upon singlet fission (direct photoexcitation), we can directly compare the differential absorption of triplet pairs to single triplets generated by photosensitization and probe differences related to electronic interactions in 
triplet pairs. In contrast to sensitization experiments, where roughly half the triplet excitons reside on a pentacene monomer and half reside on a tetracene monomer, the product of iSF is one pentacene and one tetracene triplet on the same molecule. While differences would be apparent in individual molecules populated with an individual triplet or triplet pair, the ensembles averages are similar, showing both pentacene and tetracene optical features (Figure 6a,b). Similar to our previous work on $\mathrm{BPn}^{41}$, we observe the convergence of the individual triplet spectrum (mixture of individual pentacene and individual tetracene triplets) and that of the triplet pair as the phenylene bridges are introduced to spatially separate the triplets.

We stress that despite the convergence of the photoexcitation and photosensitization transient spectra in the bridged compounds, the recombination of the triplet pair and isolated triplet remain quite distinct (Figure 6c), indicating a correlated triplet pair (multiexciton) state. Here, we denote a correlated triplet pair as ${ }^{M}(T T)$ where $M$ is the appropriate multiplicity (e.g., $M=1$ for a net singlet) in contrast to an individual triplet denoted as $T_{1}$. For example, the primary decay components of the triplet pair in PTo, PT1, and PT2 are 2.4, ns, 36 ns, and 45 ns, respectively, while individual triplets populated by sensitization in these systems decay in tens of microseconds (Figure $6 \mathrm{~d}$ ). The time scales associated with triplet pair decay are summarized in Table 1 . In transient absorption, a weaker secondary component with a longer lifetime is observed in the triplet pair decay kinetics of PT1 and PT2. This feature is discussed in detail below. Unlike the sensitized individual triplet decay, the transient spectra as a function of time do not evolve and show the same relative spectral weight for tetracene and pentacene features as a function of time (SI). Our ability to spectrally resolve concerted triplet pair decay in heterodimers allows us to explain a similar effect that was observed in symmetric dimers, where triplet pair lifetimes are orders of magnitude shorter than sensitized triplet lifetimes.

In these heterodimers, the presence of a delayed fluorescence signal allows us to further differentiate between signals originating from correlated triplet pairs and uncorrelated triplets. In our PTn compounds, correlated triplet pairs are nearly isoenergetic to the singlet, and as a result, population transfer between the singlet and triplet manifolds (Figure 7a) can occur if angular momentum conservation is satisfied. Since the triplet pairs produced by iSF are correlated into an overall singlet, ${ }^{1}(T T)$, facile coupling back to the singlet exciton via a quasi-first order process state results in a long-lived delayed fluorescence signal. The effect of this delayed fluorescence signal can be clearly seen in measurements of the photoluminescence quantum yield (Figure $7 \mathrm{~b}$ ). In contrast to analogous BPn compounds, where no delayed fluorescence is observed due to unfavorable energetics, the emission from these molecules is quite strong, with the PT2 quantum yield reaching $20 \%$ of monomeric TIPS-pentacene.

We have measured the delayed fluorescence of the correlated triplet pair using photon-counting techniques
(Figure 7c). The emission lifetimes for delayed fluorescence are identical to the primary decay component of the triplet pair lifetime as measured using nanosecond transient absorption, confirming our assignment of the state to ${ }^{1}(T T)$, a triplet pair that is correlated into an overall singlet. Electronic correlations in the spatially constrained triplet pair make the observed triplet-triplet annihilation process quasi-first order, in contrast to second order non-geminate diffusional annihilation in molecular crystals. The longer microsecond timescale component in transient absorption measurements of the PT1 and PT 2 compounds with, $\sim 5 \%$ and $20 \%$ of the total amplitude, respectively, is not present in the measurements of the fluorescence decays. As such, we assign these signals to a minority population of dark triplets, which can no longer couple back to the singlet manifold, that form in PT1 and PT2 due to an additional slow relaxation process. These species are either triplet pairs with nonsinglet spin multiplicity, i.e., ${ }^{3}(T T)$ or ${ }^{5}(T T)$, or fully localized, independent triplets $\left(2 \times T_{1}\right)$. The small population of uncorrelated triplets makes it difficult to determine their exact nature and decay dynamics using optical techniques alone. A more rigorous determination of their spin properties is necessary and is underway in our group.

From the evidence described above, we conclude that correlations exist during the primary (faster) decay component of the triplet pair. To summarize, this is based on 1) the concerted decay of features corresponding to pentacene and tetracene triplets are observed, 2) the recombination kinetics are faster in the triplet pair than in the corresponding individual triplets (Figure 6d), and 3) the primary decay component observed in transient absorption measurements corresponds to decay of a delayed fluorescence signal, indicating net singlet character. The presence of both correlated triplet pairs and uncorrelated triplets in these compounds explains the multiexponential triplet decay dynamics we have previously reported for $\mathrm{BP}_{1}$ and $\mathrm{BP}_{2}$ compounds. ${ }^{41}$ However, in the previous study we were unable to directly differentiate between triplet states because of the symmetric nature of the BPn compounds and the lack of delayed fluorescence. It is striking that despite the highly localized nature of an individual triplet, strong long-range electronic correlations exist in triplet pairs bridged by multiple phenylene spacers. This observation is especially notable in light of our observation that intramolecular triplet transport is not present in these systems, as demonstrated in Figure 5.

We note that the longer tail observed in timeresolved photoluminescence measurements of PTo results from the small amount of fluorescent impurities (TIPSpentacene monomer) which exist at a level of $<1 \%$. This impurity is visible only in fluorescence measurements because of the high sensitivity of this technique and the long lifetime of the impurity relative to the triplet pair recombination. These signals are similar to what has also been reported in bipentacene compounds. ${ }^{41}$ Impurity signals are not directly visible in PT1 and PT2 because of the longer-lived delayed fluorescence signal. 


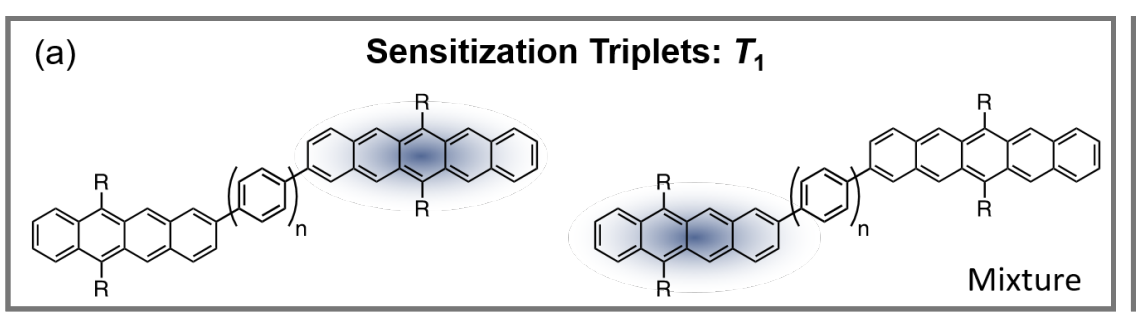

(b) Singlet Fission: ${ }^{1}(T T)$

(c)
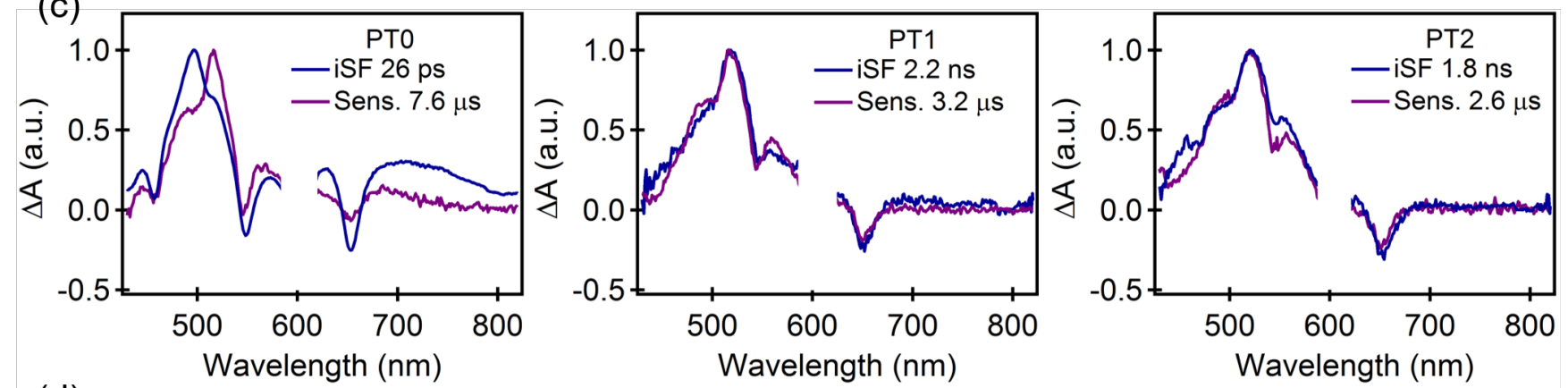

(d)
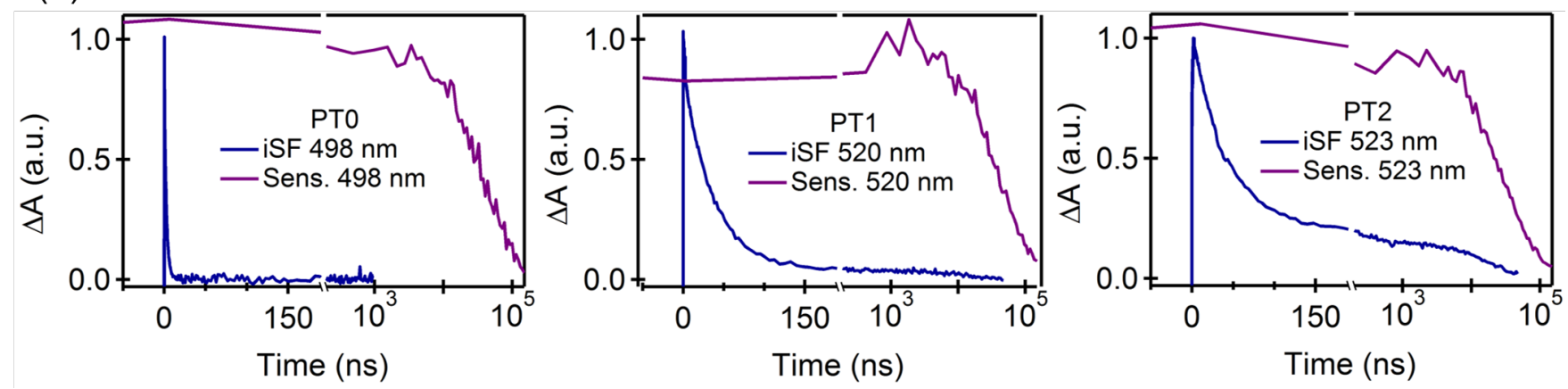

Figure 6. a) Photosensitization experiment, where triplets are populated roughly equally on pentacene and tetracene subunits, and b) singlet fission experiment where a triplet pair is populated. The spectral comparison in c) reveals that significant spectral mismatch is only observed when the subunits are directly linked (PTo), resulting in a strongly correlated triplet pair. The kinetic comparison in d) shows the lifetime of triplet pairs is shorter than sensitization-populated triplets, but can be extended by in-

\section{Analysis of Triplet Pair Recombination Processes}

The time evolution of the photoexcited singlet and correlated triplet pair populations are summarized in the diagram in Figure $7 \mathrm{a}$, and marked with their corresponding rate constants. Here we use a dark state $(D)$ to represent correlated triplet pairs that have decayed either nonradiatively back to the ground state or relaxed into uncorrelated triplets. As such, in our model $S_{0}^{\prime}$ is a modified ground state that represents only species that have decayed radiatively from the singlet. This representation allows the overall process to be modeled using a set of coupled differential equations, which were solved numerically to determine the triplet-triplet annihilation rate constant $\left(\mathrm{k}_{\text {TTA }}\right)$ (details in SI). For example, the equation governing the evolution of the correlated triplet pair is:

$$
\frac{d\left[{ }^{1}(T T)\right]}{d t}=k_{i S F}\left[S_{1}\right]-k_{T T A}\left[{ }^{1}(T T)\right]-k_{D}\left[{ }^{1}(T T)\right]
$$

The iSF rate constant $\left(\mathrm{k}_{\mathrm{iSF}}\right)$ and overall triplet pair population dynamics $\left[{ }^{1}(T T)\right]_{\mathrm{t}}$ were directly determined via global analysis of transient absorption measurements. The radiative decay constant $\left(\mathrm{k}_{\mathrm{R}}\right)$ from the $\mathrm{S}_{1}$ state was assumed to be identical to monomeric TIPS-pentacene based on previous measurements of pentaceneanthracene heterodimers. ${ }^{43}$ In our full model, we have included the effects of a small impurity contribution (at a level of $0.7 \%$ ) that is observable in photoluminescence measurements (details in SI).

The triplet-triplet annihilation rate constant $\left(\mathrm{k}_{\text {TTA }}\right)$ is uniquely determined when our solution reproduces the experimental fluorescence quantum yield, time-resolved decay measurements (proportional to the singlet population as a function of time), and the triplet pair population dynamics. In Figure $7 \mathrm{~b}$, the dotted lines are the modeled fluorescence quantum yield values (relative to TIPSpentacene), determined from $\left[S_{0}^{\prime}\right]$ at $t=\infty$, for the $\mathrm{k}_{\text {TTA }}$ values are listed in Table 1 . Similarly, the modeled populations of the singlet state (including the impurity contribution) as a function of time are plotted as the solid lines in Figure $7 \mathrm{c}$. With additional spacer length, triplet-triplet annihilation slows down with a similar scaling behavior as the singlet fission rate constants in these compounds.

The rate constants representing direct decay from the triplet pair $\left(\mathrm{k}_{\mathrm{D}}\right)$ were similarly determined from fitting. In BPn compounds, where no TTA is possible, the triplet pair decay constant is identical to $\mathrm{k}_{\mathrm{D}}$. However, in PTn, triplet-triplet annihilation and subsequent radiative decay 
modifies the overall triplet pair decay rate, such that it deviates from $k_{\mathrm{D}}$. In PT1 and $\mathrm{PT}_{2}$, the presence of TTA shortens the overall triplet pair lifetime since decay from the singlet manifold via delayed fluorescence is a viable relaxation channel. Interestingly, the opposite effect is seen in PTo. Since in this system, the singlet lifetime is longer than $\mathrm{k}_{\mathrm{D}}$, interconversion between the singlet and triplet manifolds extends the total lifetime of the triplet pair state. Because the excited singlet is longer lived than the triplet pair, this equilibration actually increases the overall triplet pair lifetime by $\sim 6 \%$ relative to the rate of $\mathrm{k}_{\mathrm{D}}$. These data are summarized in Table 1.

Table 1. Time constants for iSF, triplet-triplet annihilation, triplet pair lifetime, and direct triplet pair relaxation for the bipentacene (BPn) series ${ }^{41}$ as well as heterodimers (PTn) reported here.

\begin{tabular}{|c|c|c|c|c|}
\hline Compound & $1 / \mathrm{k}_{\mathrm{iSF}}(\mathrm{ps})$ & $1 / \mathrm{k}_{\mathrm{TTA}}(\mathrm{ps})$ & $\tau_{1(T T)}$ & $1 / \mathrm{k}_{\mathrm{D}}(\mathrm{ns})$ \\
\hline $\mathrm{PTo}$ & 0.83 & 10 & 2.4 & 2.3 \\
\hline $\mathrm{PT}_{1}$ & 18.3 & 480 & 36 & 40 \\
\hline $\mathrm{PT}_{2}$ & 640 & 9300 & 45 & 51 \\
\hline $\mathrm{BPo}_{\mathrm{BP}}$ & 0.76 & - & 0.45 & 0.45 \\
\hline $\mathrm{BP}_{2}$ & 20 & - & 16.5 & 16.5 \\
\hline
\end{tabular}

(a)
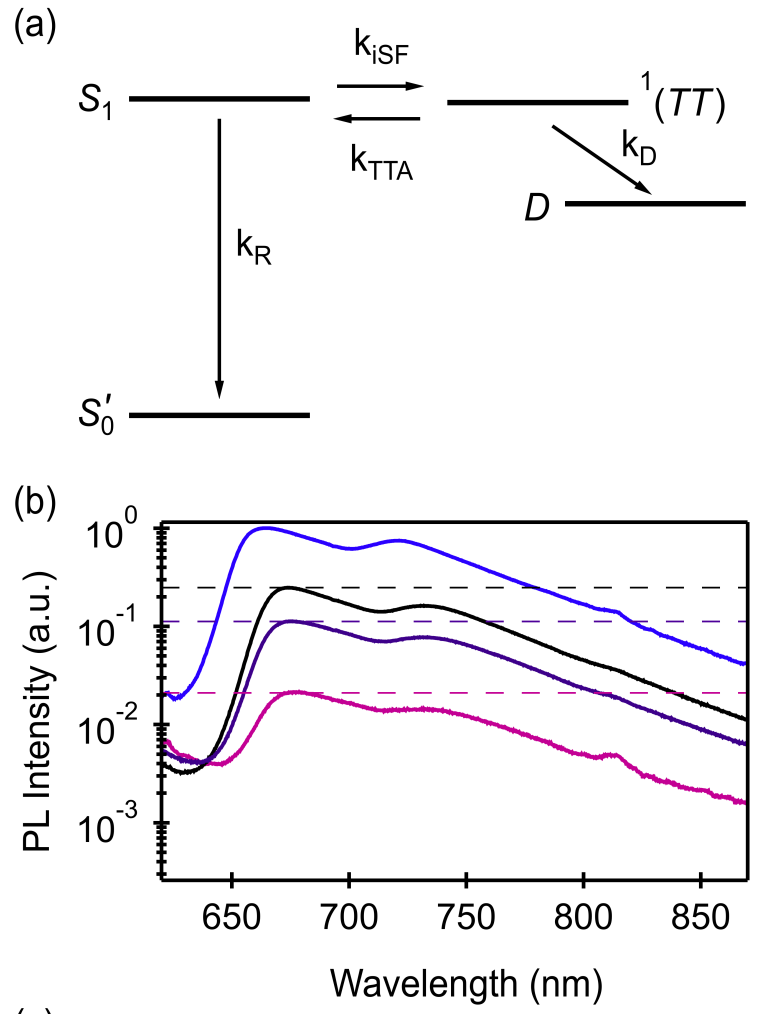

(c)

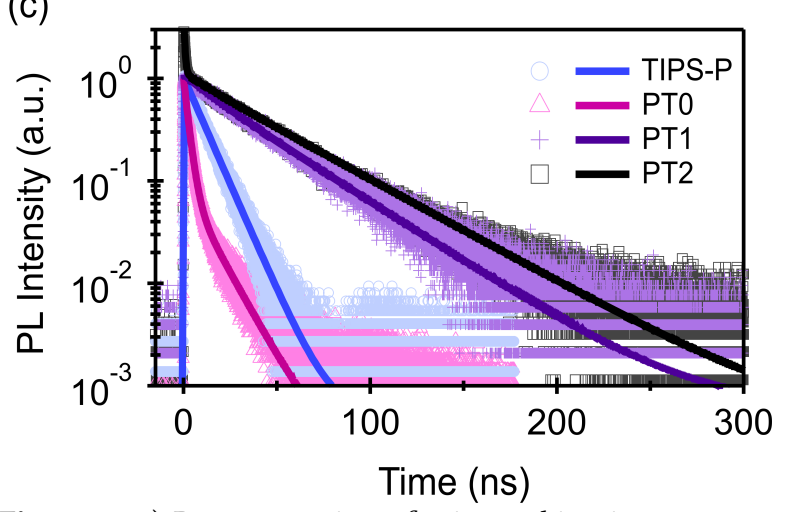

Figure 7. a) Representation of primary kinetic processes and associated rate constants governing the excited state relaxation dynamics after populating the heterodimer singlet $\left(\mathrm{S}_{1}\right)$ and triplet pair ${ }^{1}(T T)$ state, including singlet fission $\left(\mathrm{k}_{\mathrm{iSF}}\right)$, triplet-triplet annihilation $\left(\mathrm{k}_{\mathrm{TTA}}\right)$, radiative recombination $\left(k_{\mathrm{R}}\right)$ and triplet pair relaxation $\left(\mathrm{k}_{\mathrm{D}}\right)$. b) Measured photoluminescence spectra (solid lines) of PTo, PT1, and PT2 relative to TIPS-pentacene monomer $(561 \mathrm{~nm}$ excitation, chloroform as solvent). In PTo, the impurity emission decays slower than the heterodimer emission, allowing us to determine the relative concentration and decay lifetime. For heterodimers, the triplet-triplet annihilation rate constant $\left(\mathrm{k}_{\mathrm{TTA}}\right)$ is determined by matching the calculated photoluminescence quantum yield (dotted lines) to the experimental ones, using the method described in the text. c) Measured (symbols) and calculated (solid lines) fluorescence decay curves. These lifetimes correspond to the triplet pair lifetimes measured using transient absorption, indicating that delayed fluorescence is occurring.

While PT1 and PT2 have similar triplet pair lifetimes ( 36 vs. $45 \mathrm{~ns}$ ), PT2 forms a significantly larger number of uncorrelated triplets. Based on the amplitude of the long 
tail of the transient absorption triplet signal in Figure 6d, we determine that approximately $25 \%$ of triplet pairs in PT2 form uncorrelated triplets compared to only $\sim 5 \%$ in PT1. A related phenomenon is the greater similarity of the triplet pair lifetime between $\mathrm{PT}_{1}$ and $\mathrm{PT}_{2}$ as compared to $\mathrm{BP} 1$ and $\mathrm{BP}_{2}$. Based on the energy gap law, the more energetic triplet pair states in PTn should have slower nonradiative decay relative to the $\mathrm{BPn}$ series. ${ }^{43,55-57}$ While the energy gap law justifies a 3.88 times longer PTo lifetime relative to $\mathrm{BPo}, \mathrm{PT} 1$ has only a 2.44 times longer lifetime than $\mathrm{BP}_{1}$, and $\mathrm{PT}_{2}$ has a 5.4 times shorter lifetime than $\mathrm{BP}_{2}$. This effect is not well captured in our model since our analysis indicates that the overall triplet pair lifetime is only weakly sensitive to $\mathrm{k}_{\mathrm{TTA}}$ until $\mathrm{k}_{\mathrm{iSF}}$ becomes comparable to $k_{R}$. As such, the enhanced delayed fluorescence observed in PT2 does not appear to be primarily responsible for the shorter than expected triplet pair lifetime. This deviation from the energy gap law is notable and not fully accounted for by the accelerated relaxation into uncorrelated triplets.

\section{CONCLUSIONS}

We have synthesized a series of phenylene-spaced pentacene-tetracene heterodimers. These compounds undergo iSF, as demonstrated by ultrafast transient absorption spectroscopy, triplet photosensitization experiments and time-resolved PL spectroscopy. The spectral differentiation of the pentacene and tetracene ground state absorption allows us to track the spatial dynamics of the exciton population in time. In particular, the impulsive appearance of tetracene-associated features when selectively exciting low energy pentacene-associated absorption features shows that a delocalized singlet exciton is the primary product of photoexcitation. However, we find that the extent of delocalization in these asymmetric dimers is dependent on the bridge length. As the delocalization becomes minimal in the longest bridged compound, the rate of singlet fission slows considerably, suggesting delocalization is important for promoting fast and efficient singlet fission. Furthermore, we find that the primary product of intramolecular singlet fission is a correlated triplet pair (multiexciton state). This state exhibits delayed fluorescence because it is correlated into an overall singlet. Because they primarily decay via a correlated process, the tetracene and pentacene triplets decay at the same rate. However, at long times, a triplet signal remains which does not correspond to a delayed fluorescence signal. This observation allows us identify a process in which correlated triplet pairs relax into a state which is not correlated into a singlet. Overall, the novel heterodimer platform enables detailed insights into exciton correlations during singlet fission, which are not accessible with other systems.

\section{ASSOCIATED CONTENT}

Experimental methods, including details of the transient absorption spectroscopy and photosensitization experiments used to assign the triplet pair state. Details of time correlated single photon counting experiments and rate constant determination. Details and results of DFT calculations. Synthetic details for PTn compounds. This material is available free of charge via the Internet at http://pubs.acs.org.

\section{AUTHOR INFORMATION}

\section{Corresponding Author}

*E-mail lcampos@columbia.edu

*E-mail msfeir@bnl.gov

\section{Author Contributions}

All authors have given approval to the final version of the manuscript.

\section{Notes}

The authors declare no competing financial interest.

\section{ACKNOWLEDGMENT}

We thank Timothy John Harvey Hele, Eric Fuemmeler, and Nandini Ananth for useful discussions concerning the electronic structure of covalent dimers. L.M.C. acknowledges support from the Office of Naval Research Young Investigator Program (Award Nooo14-15-1-2532), ACS Petroleum Research Fund, 3M Non-Tenured Faculty Award, Arthur C. Cope Scholar Award, and Cottrell Scholar Award. S.N.S. and A. B. P. thank the NSF for GRFP (DGE 11-44155). This research used resources of the Center for Functional Nanomaterials, which is a U.S. DOE Office of Science Facility, at Brookhaven National Laboratory under Contract No. DE-SCoo12704. We are grateful to the Nuckolls lab for use of their UV-vis spectrophotometer.

\section{REFERENCES}

(1) Hanna, M. C.; Nozik, A. J. J. Appl. Phys. 2006, 100, 074510.

(2) Congreve, D. N.; Lee, J.; Thompson, N. J.; Hontz, E.; Yost, S. R.; Reusswig, P. D.; Bahlke, M. E.; Reineke, S.; Van Voorhis, T.; Baldo, M. A. Science 2013, 340, 334.

(3) Lee, J.; Jadhav, P.; Baldo, M. A. Appl. Phys. Lett. 2009, 95, 033301.

(4) Smith, M. B.; Michl, J. Annu. Rev. Phys. Chem. 2013, 64, 361.

(5) Smith, M. B.; Michl, J. Chem. Rev. 2o10, 110, 6891.

(6) Tayebjee, M. J. Y.; McCamey, D. R.; Schmidt, T. W. J. Phys. Chem. Lett. $2015,6,2367$.

(7) Tayebjee, M. J. Y.; Gray-Weale, A. A.; Schmidt, T. W. J. Phys. Chem. Lett. 2012, 3, 2749.

(8) Tayebjee, M. J. Y.; Hirst, L. C.; Ekins-Daukes, N. J.; Schmidt, T. W. J. Appl. Phys. 2010, 108, 124506.

(9) Low, J. Z.; Sanders, S. N.; Campos, L. M. Chem. Mater. 2015, 27, 5453.

(10) Monahan, N.; Zhu, X.-Y. Annu. Rev. Phys. Chem. 2015, 66, 601.

(11) Zhu, X. Acc. Chem. Res. 2013, 46, 1239.

(12) Chan, W.-L.; Berkelbach, T. C.; Provorse, M. R.; Monahan, N. R.; Tritsch, J. R.; Hybertsen, M. S.; Reichman, D. R.; Gao, J.; Zhu, X.-Y. Acc. Chem. Res. 2013, 46, 1321.

(13) Chan, W.-L.; Ligges, M.; Zhu, X. Y. Nat Chem 2012, 4, 840.

(14) Chan, W.-L.; Ligges, M.; Jailaubekov, A.; Kaake, L.; Miaja-Avila, L.; Zhu, X.-Y. Science 2011, 334, 1541.

(15) Zeng, T.; Hoffmann, R.; Ananth, N. J. Am. Chem. Soc. 2014, 136, 5755 .

(16) Zeng, T.; Ananth, N.; Hoffmann, R. J. Am. Chem. Soc. 2014, 136, 12638.

(17) Miyata, K.; Tanaka, S.; Sugimoto, T.; Watanabe, K.; Uemura, T.; Takeya, J.; Matsumoto, Y. In Ultrafast Phenomena XIX; Yamanouchi, K., Cundiff, S., de Vivie-Riedle, R., Kuwata-Gonokami, M., DiMauro, L., Eds.; Springer International Publishing: 2015; Vol. 162, p 218. 
(18) Bakulin, A. A.; Morgan, S. E.; Kehoe, T. B.; Wilson, M. W. B.; Chin, A. W.; Zigmantas, D.; Egorova, D.; Rao, A. Nature Chemistry $\mathbf{2 0 1 5}, 8,16$.

(19) Musser, A. J.; Liebel, M.; Schnedermann, C.; Wende, T.; Kehoe, T. B.; Rao, A.; Kukura, P. Nat Phys 2015, 11, 352.

(20) Busby, E.; Berkelbach, T. C.; Kumar, B.; Chernikov, A.; Zhong, Y.; Hlaing, H.; Zhu, X. Y.; Heinz, T. F.; Hybertsen, M. S.; Sfeir, M. Y.; Reichman, D. R.; Nuckolls, C.; Yaffe, O. J. Am. Chem. Soc. 2014, 136, 10654 .

(21) Berkelbach, T. C.; Hybertsen, M. S.; Reichman, D. R. J. Chem. Phys. 2013, 138, 114102.

(22) Tayebjee, M. J. Y.; Clady, R. G. C. R.; Schmidt, T. W. PCCP 2013 $15,14797$.

(23) Yost, S. R.; Lee, J.; Wilson, M. W. B.; Wu, T.; McMahon, D. P.; Parkhurst, R. R.; Thompson, N. J.; Congreve, D. N.; Rao, A.; Johnson, K.; Sfeir, M. Y.; Bawendi, M. G.; Swager, T. M.; Friend, R. H.; Baldo, M. A.; Van Voorhis, T. Nat. Chem. 2014, 6, 492.

(24) Dillon, R. J.; Piland, G. B.; Bardeen, C. J. J. Am. Chem. Soc. 2013, 135,17278

(25) Ryerson, J. L.; Schrauben, J. N.; Ferguson, A. J.; Sahoo, S. C.; Naumov, P.; Havlas, Z.; Michl, J.; Nozik, A. J.; Johnson, J. C. J. Phys. Chem. C 2014, 118, 12121.

(26) Sharifzadeh, S.; Darancet, P.; Kronik, L.; Neaton, J. B. J. Phys. Chem. Lett. 2013, 4, 2197.

(27) Bayliss, S. L.; Chepelianskii, A. D.; Sepe, A.; Walker, B. J.; Ehrler, B.; Bruzek, M. J.; Anthony, J. E.; Greenham, N. C. Phys. Rev. Lett. 2014, 112, 238701.

(28) Sternlicht, H.; Nieman, G. C.; Robinson, G. W. J. Chem. Phys. 1963, 38, 1326.

(29) Burdett, J. J.; Bardeen, C. J. J. Am. Chem. Soc. 2012, 134, 8597.

(30) Chan, W.-L.; Tritsch, J. R.; Zhu, X. Y. J. Am. Chem. Soc. 2012, 134, 18295 .

(31) Wilson, M. W. B.; Rao, A.; Johnson, K.; Gélinas, S.; di Pietro, R.; Clark, J.; Friend, R. H. J. Am. Chem. Soc. 2013, 135, 16680.

(32) Scholes, G. D. J. Phys. Chem. A 2015, 119, 12669.

(33) Trinh, M. T.; Zhong, Y.; Chen, Q.; Schiros, T.; Jockusch, S.; Sfeir, M. Y.; Steigerwald, M.; Nuckolls, C.; Zhu, X. J. Phys. Chem. C 2014, 119, 1312.

(34) Chan, W.-L.; Ligges, M.; ZhuX-Y. Nat Chem 2012, 4, 840.

(35) Stern, H. L.; Musser, A. J.; Gelinas, S.; Parkinson, P.; Herz, L. M.; Bruzek, M. J.; Anthony, J.; Friend, R. H.; Walker, B. J. Proc. Nat Acad. Sci. 2015, 112, 7656

(36) Charbr, M.; Williams, D. F. Chem. Phys. Lett. 1977, 49, 599.

(37) Geacintov, N. E.; Burgos, J.; Pope, M.; Strom, C. Chem. Phys.

Lett. 1971, 11, 504 .

(38) Busby, E.; Xia, J.; Low, J. Z.; Wu, Q.; Hoy, J.; Campos, L. M.; Sfeir, M. Y. J. Phys. Chem. B 2015, 119, 7644
(39) Busby, E.; Xia, J.; Wu, Q.; Low, J. Z.; Rong, R.; Miller, J. R.; Zhu, X.-Y.; Campos, L. M.; Sfeir, M. Y. Nat. Mater. 2014, 14, 426.

(40) Lukman, S.; Musser, A. J.; Chen, K.; Athanasopoulos, S.; Yong, C. K.; Zeng, Z.; Ye, Q.; Chi, C.; Hodgkiss, J. M.; Wu, J.; Friend, R. H.; Greenham, N. C. Adv. Funct. Mater. 2015, 25, 5452.

(41) Sanders, S. N.; Kumarasamy, E.; Pun, A. B.; Trinh, M. T.; Choi, B.; Xia, J.; Taffet, E. J.; Low, J. Z.; Miller, J. R.; Roy, X.; Zhu, X. Y.; Steigerwald, M. L.; Sfeir, M. Y.; Campos, L. M. J. Am. Chem. Soc. 2015, 137, 8965 .

(42) Zirzlmeier, J.; Lehnherr, D.; Coto, P. B.; Chernick, E. T.; Casillas, R.; Basel, B. S.; Thoss, M.; Tykwinski, R. R.; Guldi, D. M. Proc. Natl Acad. Sci. 2015, 112, 5325

(43) Sanders, S. N.; Kumarasamy, E.; Pun, A. B.; Steigerwald, M. L.; Sfeir, M. Y.; Campos, L. M. Angew. Chem. Int. Ed. 2016, 55, 3373.

(44) Pensack, R. D.; Tilley, A. J.; Parkin, S. R.; Lee, T. S.; Payne, M. M.; Gao, D.; Jahnke, A. A.; Oblinsky, D. G.; Li, P.-F.; Anthony, J. E.; Seferos, D. S.; Scholes, G. D. J. Am. Chem. Soc. 2015, 137, 6790

(45) Pensack, R. D.; Tilley, A. J.; Parkin, S. R.; Lee, T. S.; Payne, M. M.; Gao, D.; Jahnke, A. A.; Oblinsky, D.; Li, P.-F.; Anthony, J. E.; Seferos, D. S.; Scholes, G. D. J. Am. Chem. Soc. 2015, 137, 6790.

(46) Pauling, L. The Nature of the Chemical Bond; Cornell University Press: Ithaca, NY, 1972.

(47) Zimmerman, P. M.; Zhang, Z.; Musgrave, C. B. Nat Chem 2o1o, 2, 648 .

(48) Fuemmeler, E. G.; Sanders, S. N.; Pun, A. B.; Kumarasamy, E.; Zeng, T.; Miyata, K.; Steigerwald, M. L.; Zhu, X. Y.; Sfeir, M. Y.; Campos, L. M.; Ananth, N. ACS Cent. Sci. 2016, Accepted.

(49) Fudickar, W.; Linker, T. J. Am. Chem. Soc. 2012, 134, 15071 (50) Anthony, J. E.; Eaton, D. L.; Parkin, S. R. Org. Lett. 2002, 4, 15. (51) Anthony, J. E.; Brooks, J. S.; Eaton, D. L.; Parkin, S. R. J. Am. Chem. Soc. 2001, $123,9482$.

(52) Sakuma, T.; Sakai, H.; Araki, Y.; Mori, T.; Wada, T.; Tkachenko, N. V.; Hasobe, T. J. Phys. Chem. A 2016, 120, 1867.

(53) Walker, B. J.; Musser, A. J.; Beljonne, D.; Friend, R. H. Nat Chem. 2013, 5, 1019.

(54) Snellenburg, J. J. L., S. P.; Seger, R.; Mullen, K. M.; van Stokum, I. H. M. J. Stat. Soft. 2012, 49, 1

(55) Wilson, J. S.; Chawdhury, N.; Al-Mandhary, M. R. A.; Younus, M.; Khan, M. S.; Raithby, P. R.; Köhler, A.; Friend, R. H. J. Am. Chem. Soc. 2001, $123,9412$.

(56) Caspar, J. V.; Meyer, T. J. J. Phys. Chem. 1983, 87, 952.

(57) Englman, R.; Jortner, J. Mol. Phys. 1970, 18, 145.

(58) Dexter, D. L. J. Chem. Phys. 1953, 21, 836. 
Table of Contents artwork:

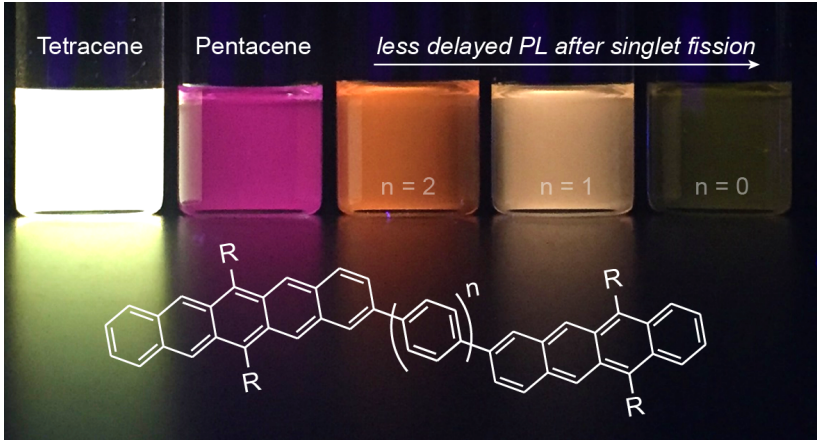

